\title{
A better prognostic assessment of the aortic root repair with valve reimplantation
}

\author{
Thierry Carrel, MD
}

\footnotetext{
From the Department for Cardiovascular Surgery, University Hospital Bern and University of Bern, Bern, Switzerland.

Disclosures: Author has nothing to disclose with regard to commercial support

Received for publication June 17, 2018; revisions received June 17, 2018; accepted for publication June 18, 2018 available ahead of print Aug 10, 2018

Address for reprints: Thierry Carrel, MD, Department for Cardiovascular Surgery, University Hospital and University of Bern, CH-3010 Bern, Switzerland (E-mail: thierry.carrel@insel.ch).

J Thorac Cardiovasc Surg 2018;156:1395-6

$0022-5223 / \$ 36.00$

Copyright (c) 2018 by The American Association for Thoracic Surgery

https://doi.org/10.1016/j.jtcvs.2018.06.053
}

The most important benefits of a valve-sparing aortic root replacement procedure are the lower incidence of thromboembolic events, the reduced risk of endocarditis, the avoidance of anticoagulation, and the potentially better durability compared with bioprostheses. Di Franco and colleagues ${ }^{1}$ are to be congratulated for their description in this issue of the Journal of a new concept, the aortic symmetry index (ASI), which they propose to predict postoperative aortic insufficiency (AI) recurrence in patients with tricuspid aortic valves undergoing the David I procedure.

Preoperatively, ASI was quantified by a formula that was suggested in a previous study. ${ }^{2}$ During a mean follow-up of 2.9 years, $5.9 \%$ had development of AI of $3+$ or more. ASI was significantly predictive of postoperative AI, in that patients in whom AI was at least $3+$ had higher ASI than did patients with no regurgitation. Di Franco and colleagues $^{1}$ concluded that-pending future, larger studies - the ASI could be used as a marker for the success of valve-sparing root replacement surgery. It appears that the study of Di Franco and colleagues ${ }^{1}$ compared only no regurgitation and regurgitation of at least $3+$; one would be interested to read more about those patients with mild to moderate aortic regurgitation after the David I procedure. It is not clear whether they were excluded from the analysis.

The length of follow-up to date is relatively short, and although some interesting results can be drawn from the current report of Di Franco and colleagues, ${ }^{1}$ some questions still remain open. Di Franco and colleagues ${ }^{1}$ affirm that "a preoperative predictor of successful VSRR [valve-sparing root replacement] in patients with AI would hold the potential to influence the timing of surgical indication." It might be interesting to analyze whether such an index might also give some information to aid the decision as to in which cases valve-sparing root replacement should be easily feasible (because of the anatomy of the aortic root and the size of each leaflet for instance) and which modifications should be performed in case of increasing ASI. Should the problem be solved at the level of the commissures

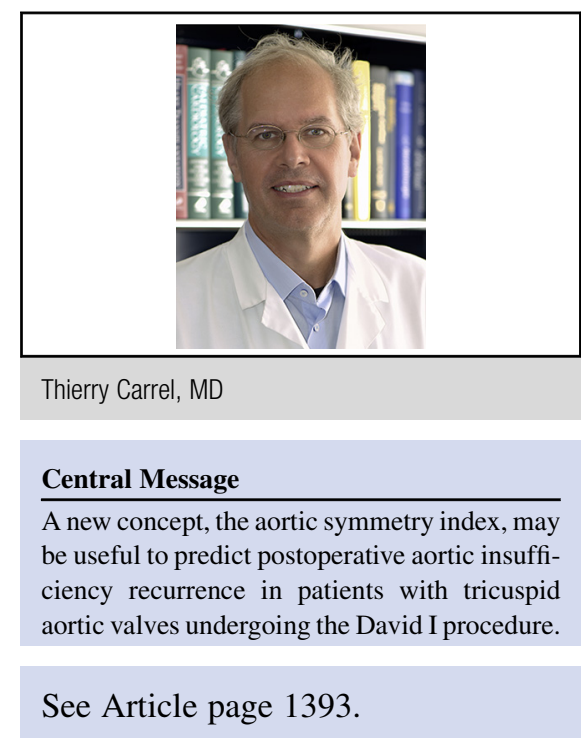

(when resuspending the aortic valve commissures into the aortic graft) or at that of the free leaflet margin (after reimplantation, in case of residual leaflet prolapse)?

Di Franco and colleagues ${ }^{1}$ examined the influence of ASI on midterm to long-term function of the reimplanted aortic valve. It might be interesting, however, to know their opinion regarding the use of such an index during intraoperative TEE at the end of the procedure and, in cases of residual AI, to analyze the mechanisms and the options to improve the function of the native valve in the aortic graft, to mimic the preoperative native anatomy as closely as possible.

It remains unknown in the article of Di Franco and colleagues $^{1}$ whether early AI recurrence might be produced by the same mechanisms as late AI recurrence after the David operation. With regard to this aspect, development of postoperative regurgitation would be a time-dependent event, and therefore it would better be analyzed in time-depend models, such as the Cox hazard model.

Severe asymmetry of the aortic valve makes a David procedure more challenging, but in the majority of cases, this can be simply addressed by leaflet plication, so long as leaflet tissue is amendable to this modification. Any technique for leaflet adjustment thus may interact between ASI and postoperative regurgitation. Such an interaction would be interesting to know about, because additional cusp interventions have been associated with increased recurrence of aortic regurgitation, at least in some series. 


\section{Reference}

1. Di Franco A, Rong LQ, Munja M, Weinsaft JW, Kim J, Sturla F, et al. Aortic symmetry index: initial validation of a novel preoperative predictor of recurrent aortic insufficiency after valve-sparing aortic root reconstruction. J Thorac Cardiovasc Surg. 2018;156:1393-4.
2. Gaudino M, Di Franco A, Weltert L, Benedetto U, Lau C, Gambardella I, et al. The role of neo-sinus reconstruction in aortic valve-sparing surgery. J Card Surg. 2017; 32:328-33. 